\title{
Flora of the Region Between Ayaş, Kazan and Yenikent (Ankara/Turkey)*
}

\author{
Sanem AKDENIZ ŞAFAK ${ }^{1 * *}$, Osman KETENOĞLU ${ }^{1}$ \\ ${ }^{1}$ Ankara Üniversitesi, Fen Fakültesi, Biyoloji Bölümü, Ankara
}

\author{
Received (Geliss): 29.06.2015
}

\author{
Accepted (Kabul): 17.11.2015
}

\begin{abstract}
In this research, the flora of the area, northwest of Ankara, among Ayaş, Kazan and Yenikent was studied. This area is within the borders of Irano-Turanian phytogeographic region and also in A4 square according to the grid system applied for the Flora of Turkey by Davis. About 500 plant samples were collected from the area between July 2007-June 2009. 268 taxa belonging to 149 genera in 37 families were determined. The richest family and the richest genus of the research area are Asteraceae and Alyssum respectively. 37 taxa of the collected plants were endemic and its ratio to total is $13.8 \%$. The distribution of species according to the phytogeographical regions is as follows; Irano-Turanian 17.2\%, Mediterranean and East Mediterranean 5.2\%, Euro-Siberian and Euxine 4.12\%, widespread or unknown $73.41 \%$.
\end{abstract}

Key Words: Flora, Ayaş, Kazan, Yenikent, Ankara, Turkey

\section{Ayaş, Kazan, Yenikent Arasında Kalan Bölgenin Florası (Ankara/Türkiye)}

ÖZET: Bu çalışmada, Ankara'nın kuzeybatısında yer alan, Ayaş, Kazan ilçeleri ve Sincan ilçesine bağlı bir belde olan Yenikent arasında kalan bölgenin florası araştırılmıştır. Temmuz 2007 ve Haziran 2009 tarihleri arasında yapılan arazi çalışmaları sonucunda yaklaşık 500 bitki örneği toplanmıştır. Bu örneklerin incelenmesi sonucunda, 37 familya, 149 cinse ait 268 takson tespit edilmiştir. Bu taksonların 37'si Endemik olup endemizm oranı \% 13.8'dir. Araştırma alanındaki en zengin familya Asteraceae ve en zengin cins Brassicaceae familyasından Alyssum olarak belirlenmiştir. Taksonların fitocoğrafik bölgelere göre dağılımları ve oranları şöyledir: İran-Turan \%17.2; Akdeniz ve Doğu Akdeniz \%5.2; Avrupa-Sibirya ve Öksin \%4.12; çok bölgeli veya bilinmeyen \%73.4.

Anahtar Kelimeler: Flora, Ayaş, Kazan, Yenikent, Ankara, Türkiye

\section{INTRODUCTION}

Turkey has different vegetation types and a rich flora because of its geographic location, geomorphologic structure, various soil types and climatic diversity. These exceptions create a rich floristic diversity in Turkey. Central Anatolia, especially city of Ankara is ranked among important areas, it terms of both floristic diversity and phytogeography. The study area, the region among Ayaş, Kazan and Yenikent, is located in the northwest of Ankara (Fig 1) and phytogeographically belongs to the Irano-Turanien region. It lies within square A4 according to grid system of Davis (1965-1985) (Fig 2.). The altitudinal range of the area changes between 700 and 1100 meters. The climate of the area is semi-arid-sub-very cold Mediterranean climate according to Emberger's pluviothermique quotient (Akman 2010). Antropogenic effects on environment such as pollution, urbanization, globalization are growing day by day, especially in the vicinities of big cities and damage naturel habitats and restraint the biodiversity of flora. To detect these destructions, floristic researches in cities or their environs that are vulnerable to such peril have been conducted in the last decades in Turkey.

Some floristic studies carried out in the vicinities of the area are as fallows:

- Flora of the Region among Ayaş, Çanıllı village and Kazan (Yavuz 1992)

- Floristic Research of Step Formations Spreading among Taşpınar, İncek, Tuluntaş villages (Ankara) (Yağcı 1994)

- A Study on Flora of Kirmir Rill (Kocaçay) Valley (Güdül-Ankara) (Tarıkahya 2003)

- A Study on Flora of Hacikadin Valley (Ankara/Turkey) (Yeşilyurt et al. 2008)

- $\quad$ Flora of Kıbrıs Köyü Valley (Mamak-Ankara) (Aslan et al. 2009)

In accordance with all these reasons, there are numerous purposes for carrying out this study and choosing this area. First of all, research area have not been thoroughly studied so far and it has richness. It is also aimed to rummage out the details which contribute to reveal the floristic richness of Turkey.

\footnotetext{
*Article part of Msc thesis

**Corresponding author: Akdeniz Şafak, S., sanem.akdeniz@ hotmail.com
} 


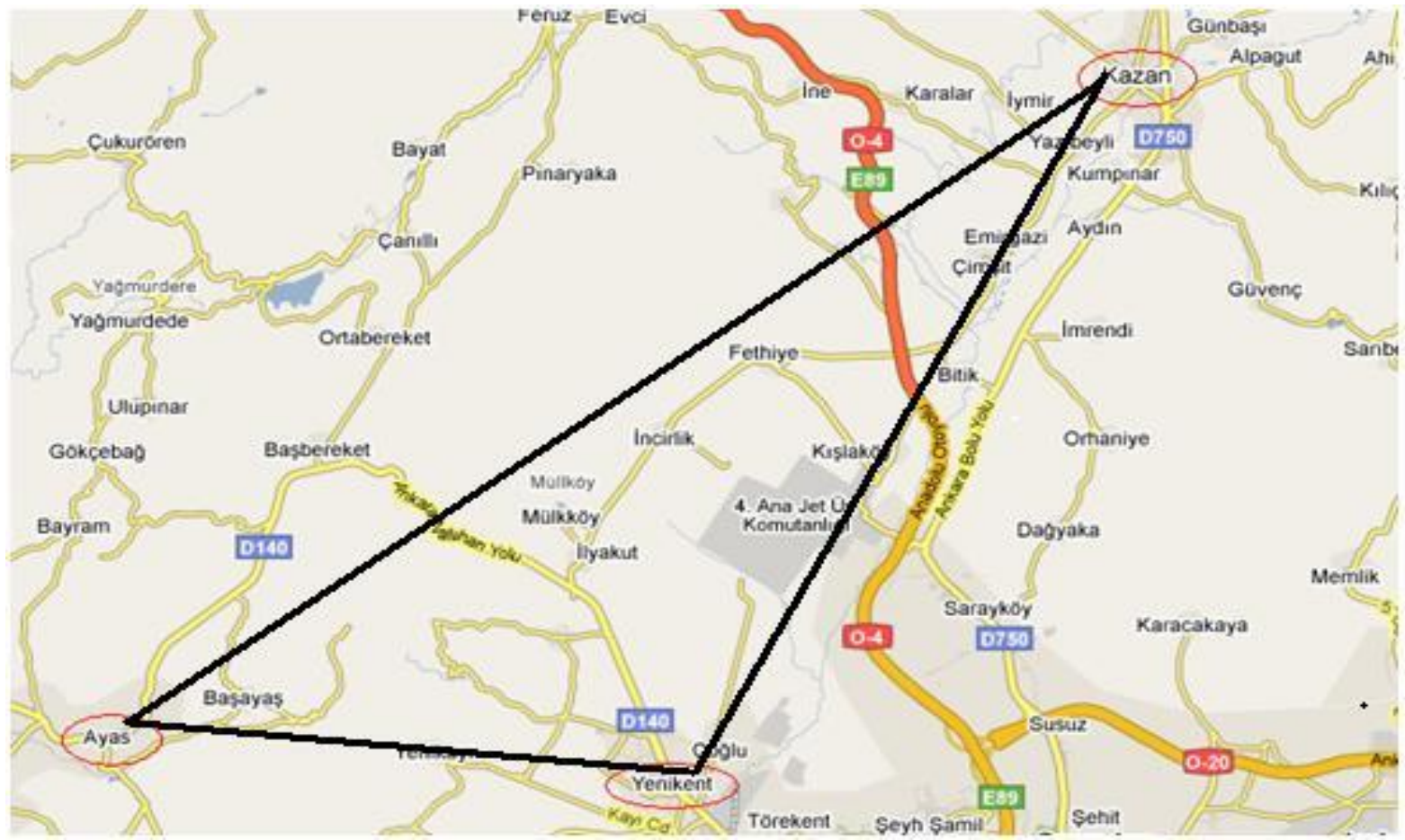

Figure 1. Map of the study area (triangle)

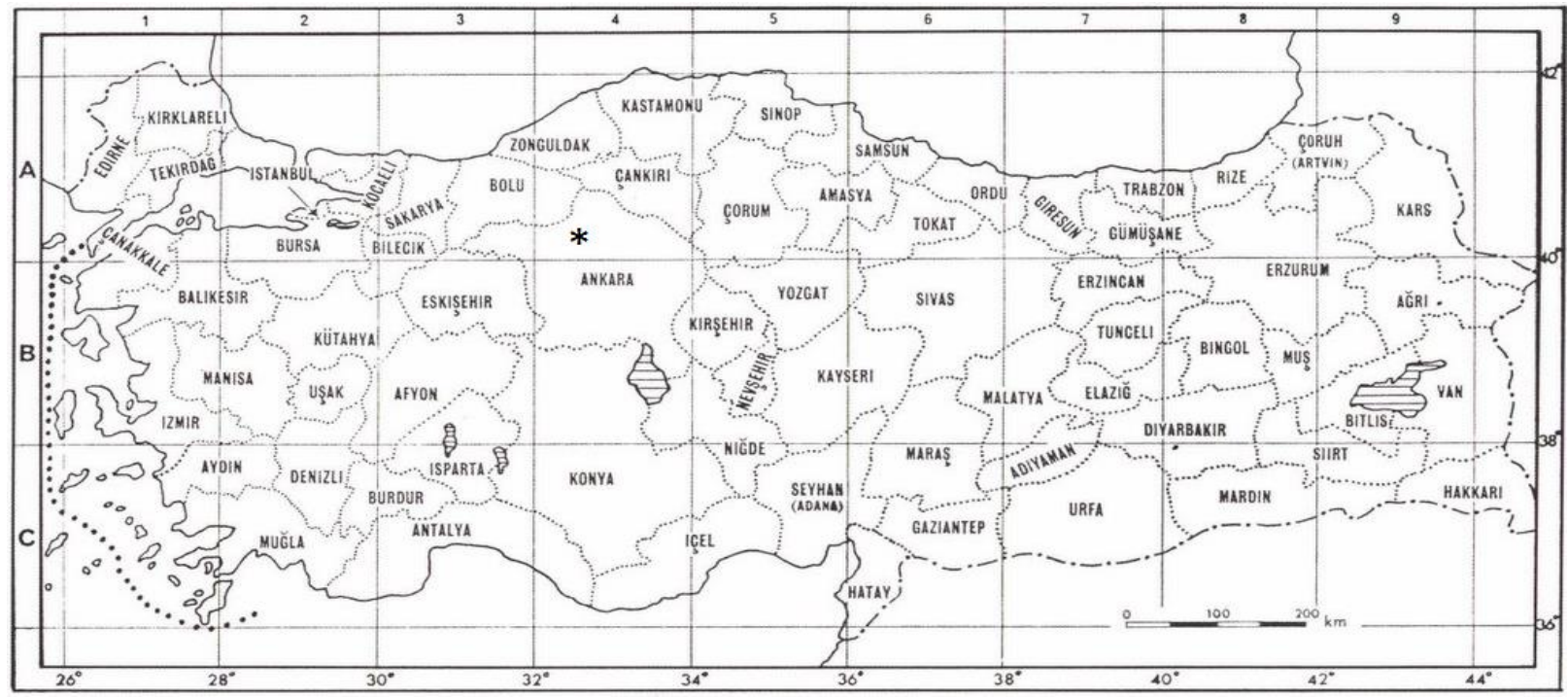

Figure 2. The location of the area according to grid system of Davis (1965-1985): A4

\section{MATERIAL and METHODS}

The research materials are plant specimens collected from the area at different vegetation periods (flowering, fruiting) between the years 2007 and 2009. The collected plants were prepared as herbarium materials and the identified. Endemism and conservation status of species were detailed by using International Union for Conservation of Nature in Red Data Book of Turkish Plants (Ekim et al. 2000) and the changes and the regulations in 2014 (IUCN Standarts and Petitions
Subcommittee Guidelines for Using the IUCN Red List Categories and Criteria 2014).

Identified plant taxa are listed according to Flora of Turkey (Davis 1965-1985, Davis 1988) and the following informations were added to each taxa: the names and authors of species, subspecies and varieties, locality, habitat, altitude, date, collector's name and number of plants; respectively, phytogeographic distribution, endemizm, conservation status. And also a dictionary (Baytop 1998), some systematic and books about plant biology (Donner 1990, Küçüker 1998, 
Kilınç et al. 2006, Seçmen et al. 2008) and some articles (Akman et al. 1979, Erik et al. 1996, Eraslan 2001) were used for identification.

The abbreviations used in the text are as follows:

\begin{tabular}{|c|c|}
\hline S. Akdeniz & $\begin{array}{l}\text { Sanem AKDENİZ } \\
\text { (collector's name) }\end{array}$ \\
\hline K & Kazan \\
\hline A & Ayaş \\
\hline Y & Yenikent \\
\hline IFN & $\begin{array}{l}\text { Provincial Directorate of } \\
\text { Environment and Forests } \\
\text { Ilyakut Forest Nursery }\end{array}$ \\
\hline
\end{tabular}

\section{RESULTS}

In the field studies carried out between July 2007 June 2009 were collected about 500 plant samples. As a result of the identification of these plant samples were determined as 268 taxa, including 37 families. Number of endemic taxa is $37(13.8 \%)$. According to The IUCN Red List Categories, distributions of these 37 endemic taxa and the names of these plants and the categories are given Table 1. The phytogeographical distribution of the taxa in the study area and their rates are as follows; Irano-Turanian 17.2\%, Mediterranean and East Mediterranean 5.2\%, Euro-Siberian and Euxine 4.12\%, Widespread or unknown $73.41 \%$. Irano-Turanian elements are the richest ones due to the location of the area.

The richest families and their numbers in the research area are as following: Asteraceae 45, Poaceae 18, Papaveraceae 12, Brassicaceae 42, Lamiaceae 17, Ranunculaceae 12, Fabaceae 28, Scrophulariaceae 12.

Table 1. The names of endemic taxa and The IUCN Red List Categories

Names of the endemic taxa

The IUCN Red List Categories

Consolida hellespontica (Boiss.) Chater EN

Glaucium grandiflorum Boiss. \& Huet. var. torquatum Cullen

Papaver commutatum Fisch. \& Mey. subsp. euxinum Kadereit

EN

Hesperis balansae Fourn. subsp. balansae Fourn.

EN

Aethionema capitatum Boiss. \& Bal

Alyssum blepharocarpum Dudley \& Hub. - Mor.

Alyssum hirsutum Bieb. var. caespitosum Dudley.

Trigonella rostrata (Boiss. \& Bal.) Boiss.

Causinia iconica Hub. - Mor.

EN

Centaurea paphlagonica (Bornm.) Wagenitz

Consolida glandulosa (Boiss. \& Huet) Bornm.

Ranunculus reuterianus Boiss.

Thlaspi violascens Boiss.

Alyssum paphlagonicum (Hausskn.) Dudley

NT

Erysimum thyrsoideum Boiss. subsp. ponticum (Hausskn. \& Bornm.) Cullen

NT

NT

Erodium absinthoides Willd. subsp. absinthoides

Astragalus lycius Boiss.

Astragalus xylobasis Freyn. \& Bornm. var. angustus (Freyn. \& Sint.) Freyn. \& Bornm.

Astragalus hirsutus Vahl.

Astragalus humillimus Freyn. \& Sint

Astragalus sigmoideus Bunge.

Onobrychis armena Boiss. \& Huet.

Helichrysum noeanum Zahn.

Achillea phrygia Boiss. \& Bal.

Cirsium leucopsis DC.

Crepis macropus Boiss. \& Heldr.

Onosma tauricum Pallas ex Willd. var. brevifolium DC.

Verbascum cheiranthifolium Boiss. var. asperulum (Boiss.) Murb.

Linaria corifolia Desf.

NT

NT

LC

$\mathrm{LC}$

$\mathrm{LC}$

$\mathrm{LC}$

LC

$\mathrm{LC}$

$\mathrm{LC}$

LC

Digitalis lamarckii Ivan.

Veronica multifida $\mathrm{L}$.

Phlomis armeniaca Willd.

Wiedemannia orientalis Fisch. \& Mey.

Stachys cretica L. subsp. anatolica Rech.

Salvia cryptantha Montbret \& Aucher

$\mathrm{LC}$

$\mathrm{LC}$

LC

$\mathrm{LC}$

$\mathrm{LC}$

LC

LC

$\mathrm{LC}$

LC

$\mathrm{LC}$

$\mathrm{LC}$

LC

LC

$\mathrm{LC}$

Asperula stricta Boiss. subsp. latibracteata (Boiss.) Ehr.

Bellevalia clusiana Griseb.

$\mathrm{LC}$

$\mathrm{LC}$

$\mathrm{LC}$

$\mathrm{LC}$

$\mathrm{LC}$ 
Table 2. Comparison of the number of total taxa with the others carried out neighbour areas

\begin{tabular}{clr}
\hline $\begin{array}{l}\text { Study code } \\
\text { number* }\end{array}$ & Name of study (Author and Year) & $\begin{array}{r}\text { Number of } \\
\text { taxa }\end{array}$ \\
\hline S1 & Flora of the Region among Ayaş, Kazan and Yenikent (Ankara/Turkey) & 268 \\
\hline S2 & Flora of the Region among Ayaş, Çanıllı Köyü, Kazan (Yavuz 1992) & 203 \\
\hline S3 & A Study on Flora of Hacıkadın Valley (Ankara/Turkey) (Yeşilyurt et al. 2008) & 343 \\
\hline S4 & Flora of Kıbrıs Köyü Valley (Mamak-Ankara) (Aslan et al. 2009) & 624 \\
\hline S5 & $\begin{array}{l}\text { A Study on Flora of Kirmir Rill (Kocaçay) Valley (Güdül-Ankara) (Tarıkahya } \\
\text { 2003) }\end{array}$ & 452 \\
\hline S6 & $\begin{array}{l}\text { Floristic Research of Step Formations Spreading among Taşpınar, İncek, } \\
\text { Tuluntaş Villages (Ankara) (Yağcı 1994) }\end{array}$ & 276 \\
\hline
\end{tabular}

*Studies, in the table, will be mentioned with their "study code number" in the text.

\section{DISCUSSION}

Results of the study have been compared with the others carried out neighbour areas. These studies are showed at Table 2.

Variability on the taxa number is depends on size of research area, dominance status of step vegetation, the presence of deep valley and antropogenic effects of the area.

Phytogeographical distribution, endemism structure and the families with the most taxa have been compared with the other studies carried out neighbour areas (respectively: Table 3, Table 4, Table 5).

Table 3. Comparison of the flora of research area with the studies carried out neighbour areas from the phytogeographical point of view

\begin{tabular}{|c|c|c|c|c|c|c|c|c|c|c|c|c|c|}
\hline \multicolumn{2}{|c|}{ Study code number } & \multicolumn{2}{|l|}{ S1 } & \multicolumn{2}{|l|}{ S2 } & \multicolumn{2}{|l|}{ S3 } & \multicolumn{2}{|l|}{ S4 } & \multicolumn{2}{|l|}{ S5 } & \multicolumn{2}{|l|}{ S6 } \\
\hline \multicolumn{2}{|c|}{$\mathrm{N}$ : Number of taxa, R: Rate $(\%)$} & $\mathrm{N}$ & $\mathrm{R}$ & $\mathrm{N}$ & $\mathrm{R}$ & $\mathrm{N}$ & $\mathrm{R}$ & $\mathrm{N}$ & $\mathrm{R}$ & $\mathrm{N}$ & $\mathrm{R}$ & $\mathrm{N}$ & $\mathrm{R}$ \\
\hline \multirow{4}{*}{ 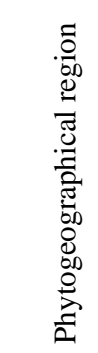 } & Irano - Turanian & 49 & 18.3 & 50 & 24.6 & 56 & 16.3 & 127 & 20.3 & 78 & 17.3 & 94 & 35.05 \\
\hline & $\begin{array}{l}\text { Mediterranean / East } \\
\text { Mediterranean }\end{array}$ & $\begin{array}{l}11 / \\
4\end{array}$ & 5.6 & 13 & 6.4 & 31 & 9.0 & 50 & 8.0 & 46 & 10.2 & 13 & 4.71 \\
\hline & $\begin{array}{l}\text { Euro - Siberian / } \\
\text { Euxine }\end{array}$ & $\begin{array}{l}10 / \\
2\end{array}$ & 4.5 & 11 & 5.4 & 30 & 8.7 & 67 & 10.7 & 43 & 9.5 & 6 & 2.17 \\
\hline & $\begin{array}{l}\text { Widespread or } \\
\text { unknown }\end{array}$ & 192 & 71.6 & 129 & 63.6 & 226 & 65.8 & 380 & 61 & 285 & 63 & 163 & 58.07 \\
\hline \multicolumn{2}{|l|}{ Total } & 268 & 100 & 203 & 100 & 343 & 100 & 624 & 100 & 452 & 100 & 276 & 100 \\
\hline
\end{tabular}

Table 4. Comparison of the flora of research area with the studies carried out neighbour areas from the endemism point of view

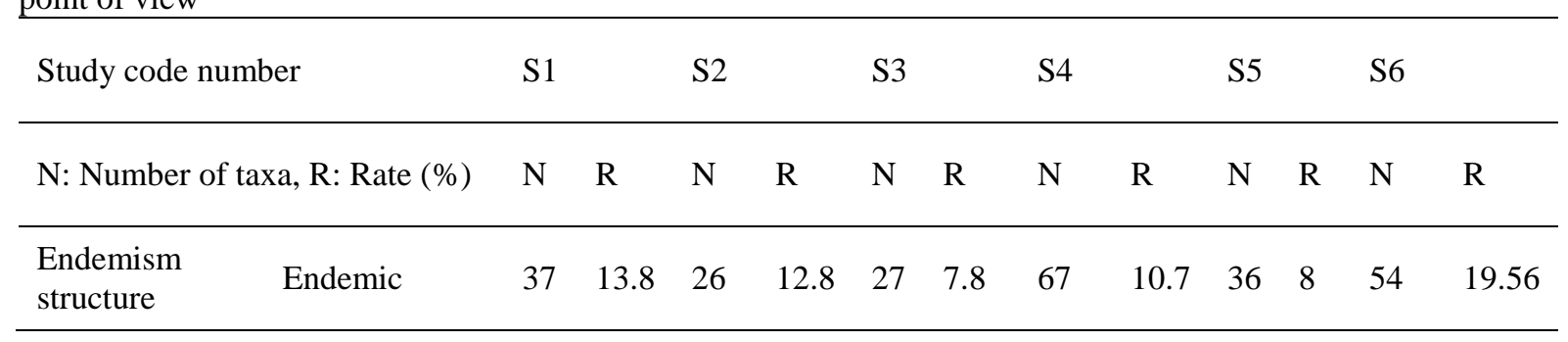


Table 5. Comparison of largest families in study area with the studies carried out near areas

\begin{tabular}{|c|c|c|c|c|c|c|}
\hline $\begin{array}{l}\text { Study } \\
\text { code } \\
\text { number }\end{array}$ & S1 & S2 & S3 & S4 & S5 & S6 \\
\hline \multirow{9}{*}{ 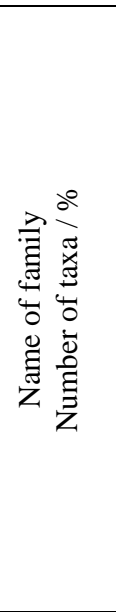 } & $\begin{array}{l}\text { Asteraceae } \\
45 / 16.8\end{array}$ & $\begin{array}{l}\text { Asteraceae } \\
26 / 12.7\end{array}$ & $\begin{array}{l}\text { Asteraceae } \\
40 / 11.6\end{array}$ & $\begin{array}{l}\text { Asteraceae } \\
87 / 13.9\end{array}$ & $\begin{array}{l}\text { Asteraceae } \\
43 / 9.5\end{array}$ & $\begin{array}{l}\text { Fabaceae } \\
38 / 13.8\end{array}$ \\
\hline & $\begin{array}{l}\text { Brassicaceae } \\
42 / 15.7\end{array}$ & $\begin{array}{l}\text { Fabaceae } \\
20 / 9.8 \\
\end{array}$ & $\begin{array}{l}\text { Fabaceae } \\
37 / 10.7 \\
\end{array}$ & $\begin{array}{l}\text { Fabaceae } \\
53 / 8.4\end{array}$ & $\begin{array}{l}\text { Brassicaceae } \\
40 / 8.9\end{array}$ & $\begin{array}{l}\text { Asteraceae } \\
36 / 13.0 \\
\end{array}$ \\
\hline & Fabaceae & Lamiaceae & Lamiaceae & Poaceae & Fabaceae & Lamiaceae \\
\hline & $28 / 10.5$ & $18 / 8.8$ & $27 / 7.8$ & $46 / 7.3$ & $37 / 8.2$ & $25 / 9.0$ \\
\hline & $\begin{array}{l}\text { Poaceae } \\
18 / 6.72\end{array}$ & $\begin{array}{l}\text { Boraginaceae } \\
15 / 7.3\end{array}$ & $\begin{array}{l}\text { Poaceae } \\
23 / 6.7\end{array}$ & $\begin{array}{l}\text { Lamiaceae } \\
45 / 7.2\end{array}$ & $\begin{array}{l}\text { Lamiaceae } \\
35 / 7.8\end{array}$ & $\begin{array}{l}\text { Poaceae } \\
20 / 7.0\end{array}$ \\
\hline & $\begin{array}{l}\text { Lamiaceae } \\
17 / 6.34\end{array}$ & $\begin{array}{l}\text { Poaceae } \\
13 / 6.8\end{array}$ & $\begin{array}{l}\text { Brassicaceae } \\
22 / 6.4\end{array}$ & $\begin{array}{l}\text { Brassicaceae } \\
35 / 5.6\end{array}$ & $\begin{array}{l}\text { Poaceae } \\
29 / 6.5\end{array}$ & $\begin{array}{l}\text { Brassicaceae } \\
19 / 6.9\end{array}$ \\
\hline & $\begin{array}{l}\text { Ranunculaceae } \\
12 / 4.5\end{array}$ & $\begin{array}{l}\text { Brassicaceae } \\
12 / 6.4\end{array}$ & $\begin{array}{l}\text { Boraginaceae } \\
19 / 5.5\end{array}$ & $\begin{array}{l}\text { Rosaceae } \\
33 / 5.2\end{array}$ & $\begin{array}{l}\text { Boraginaceae } \\
24 / 5.3\end{array}$ & $\begin{array}{l}\text { Caryophyllaceae } \\
14 / 5.0\end{array}$ \\
\hline & $\begin{array}{l}\text { Papaveraceae } \\
12 / 4.5\end{array}$ & $\begin{array}{l}\text { Caryophyllaceae } \\
8 / 3.9\end{array}$ & $\begin{array}{l}\text { Caryophyllaceae } \\
18 / 5.2\end{array}$ & $\begin{array}{l}\text { Apiacea } \\
31 / 4.9 \\
\end{array}$ & $\begin{array}{l}\text { Apiaceae } \\
21 / 4.6 \\
\end{array}$ & $\begin{array}{l}\text { Apiaceae } \\
14 / 5.0\end{array}$ \\
\hline & $\begin{array}{l}\text { Scrophulariacea } \\
\text { e } 12 / 4.5\end{array}$ & $\begin{array}{l}\text { Apiaceae } \\
8 / 3.9\end{array}$ & $\begin{array}{l}\text { Rosaceae } \\
16 / 4.6\end{array}$ & $\begin{array}{l}\text { Boraginaceae } \\
26 / 4.1\end{array}$ & $\begin{array}{l}\text { Rosaceae } \\
19 / 4.2\end{array}$ & $\begin{array}{l}\text { Boraginaceae } \\
11 / 4.0\end{array}$ \\
\hline Total & 268 & 203 & 343 & 624 & 452 & 276 \\
\hline
\end{tabular}

Phytogeographical distribution of the study and the others shows parallelism. In all of the studies, IranoTuranian elements occupy the first rank due to the location of all areas in the same phytogeographical region. In respect to endemism S6 coded study has the highest rate with $19.56 \%$ and followed by this study with $13.8 \%$. In terms of the families with the most taxa, in the five of studies (included our study), Asteraceae is the richest family as is in the flora of Turkey. And the other richest families are Fabaceae, Brassicaceae, Poaceae, Boraginaceae, Lamiaceae, Apiaceae, Scrophulariaceae, Ranunculaceae, Caryophyllaceae, Rosaceae, Liliaceae. These are known as the richest families in Turkey. All studies exhibite similarities about having the most taxa.

\section{REFERENCES}

Akman, Y., Ketenoğlu, O. 1979. Contribution à Létude De La Flore Des Montagnes D’Ayaş. Communications De La Faculté Des Sciences De L'université D'Ankara, Serie C2, 23, Supplement: 2. 1-38.

Akman, Y. 2011. Biyocoğrafya. Palme Yayınları, Ankara, $449 \mathrm{~s}$.

Aslan, S., Vural, M. 2009. Flora of Kibris Köyü Valley (Mamak-Ankara-Turkey). Biodicon 2/3: 34-64.

Baytop, A. 1998. İngilizce - Türkçe Botanik Kılavuzu. İstanbul Üniversitesi, Eczacılık Fakültesi, Yayın No: $70,375 \mathrm{~s}$.

Davis, P.H. 1965-1985. Flora of Turkey and the East Aegean Islands. Volume 1-9, University Press., Edinburgh.

Davis, P. H., Mill, R.R., Tan, K. 1988. Flora of Turkey and the East Aegean Islands (Supplement). Volume 10, University Press., Edinburgh.

Donner, J. 1990. Distribution Maps to P. H. Davis, Flora of Turkey. Volume 1-10, Linzer Biol. Breitr, 22-2381515, 135 s, Linz.
Ekim, T., Koyuncu, M., Duman, H., Aytaç, Z., Adıgüzel, N. 2000. Türkiye Bitkileri Kırmızı Kitabı (Eğrelti ve Tohumlu Bitkiler). Van Yüzüncüyıl Üniversitesi, Türkiye Tabiatını Koruma Derneği, 246 s.

Eraslan, Z. 2001. Oyaca, İkizce, Germeyik Köyleri Arasında Kalan Bölgenin Florası. Gazi Üniversitesi, Fen Bil. Ens., Yüksek Lisans Tezi, 81 s, Ankara.

Erik, S., Akaydın, G. 1996. A4 Karesi (Ankara) İçin Yeni Kayıtlar. Hacettepe Üniversitesi, Eğitim Fakültesi Dergisi, 12: 211-213.

IUCN, 2014. Standarts and Petitions Subcommittee Guidelines for Using the IUCN Red List Categories and Criteria. Version 11. Prepared by the Standards and Petitions Subcommittee of the IUCN Species Survival Commission. February 2014.

Kılınç, M., Kutbay H. G., Yalçın, E., Bilgin, A. 2006. Bitki Ekolojisi ve Bitki Sosyolojisi Uygulamaları, Yayın No: 394, $362 \mathrm{~s}$.

Küçüker, O. 1998. Bitki Morfolojisi I. Kapalı Tohumlu Bitkiler. İstanbul Üniversitesi, Fen Fakültesi Yayınları, Yayın No: 248, $207 \mathrm{~s}$.

Seçmen, Ö., Gemici, Y., Leblebici, E., Görk, G., Bekat, L. 2008. Tohumlu Bitkiler Sistematiği. Ege Üniversitesi, Fen Fakültesi Kitaplar Serisi, No: 116, 432 s.

Tarıkahya, B. 2003. Kirmir Çayı (Kocaçay) Vadisi (GüdülAnkara) Florası Üzerine Bir Araştırma. Hacettepe Üniversitesi, Fen Bil. Ens., Yüksek Lisans Tezi, 183 s.

Yağcı, A. 1994. Taşpınar, İncek, Tuluntaş (Ankara) Köyleri Arasında Yayılış Gösteren Step Formasyonlarının Floristik Yönden Araştırılması. Gazi Üniversitesi, Fen Bil. Ens., Yüksek Lisans Tezi, 78 s.

Yavuz, H. 1992. Ayaş, Çanıllı Köyü, Kazan Arasında Kalan Bölgenin Florası. Ankara Üniversitesi. Fen Bil. Ens., Yüksek Lisans Tezi, 71 s.

Yeşilyurt, E. B. Kurt, L., Akaydın, G. 2008. A Study on Flora of Hacikadın Valley (Ankara). Biodicon, 1/2: 2552. 


\section{PINACEAE}

\section{SPERMATOPHYTA} GYMNOSPERMAE

1. Pinus nigra Arn. subsp. pallasiana (Lamb.) Holmboe, Y, forest, 850 m, 23.04.2008, S. Akdeniz 1074.

2. CUPRESSACEAE

2. Juniperus oxycedrus L. subsp. oxycedrus L., A, Mountain pass of Ayaş, forest gaps, 1060 m, 15.05.2009, S. Akdeniz 1279.

\section{RANUNCULACEAE}

\section{ANGIOSPERMAE DICOTYLEDONAE}

3. Nigella segetalis Bieb., A, field edge, 910 m, 28.05.2008, S. Akdeniz 1095.

4. Consolida orientalis (Gay) Schröd

A, cultivated areas, 850 m, 28.05.2008, S. Akdeniz 1347a., Y, roadside, 780 m, 31.05.2009, S. Akdeniz $1347 \mathrm{~b}$.

5. C. regalis S. F. Gray subsp. paniculata (Host) Soo var. paniculata Host, K, step, 900 m, 08.07.2007, S. Akdeniz 1002., K, cultivated areas, 900 m, 07.08.2007, S. Akdeniz 1049., A, step, 960 m, 25.06.2008, S. Akdeniz 1349.

6. C. glandulosa (Boiss. \& Huet) Bornm., A, cultivated land, $960 \mathrm{~m}, 29.06 .2009$, S. Akdeniz 1348. Irano Turanian. Endemic. LC.

7. C. hellespontica (Boiss.) Chater, K, step, 780 m, 08.07.2007, S. Akdeniz 1003. Endemic. EN.

8. Adonis aestivalis L. subsp. aestivalis L., Y, cultivated area, 855 m, 23.04.2008 S. Akdeniz 1090., A, rocky slopes, 890 m, 28.05.2008, S. Akdeniz 1350.

9. A. flammea Jacq., A, step, 869 m, 28.05.2008, S. Akdeniz 1351a., Y, IFN, 800 m, 16.04.2009, S. Akdeniz 1351b.

A, Mountain pass of Ayaş, stony slopes, 1060 m, 15.05.2009, S. Akdeniz 1352c.

10. Ranunculus oxyspermus Wild., A, field, 800 m, 28.05.2008, S. Akdeniz 1093.

11. R. reuterianus Boiss., Y, stony slopes, 925 m, 23.04.2008, S. Akdeniz 1064. Endemic. LC.

12. $R$. illyricus L. subsp. illyricus L., A, cultivated areas, 925 m, 28.05.2008, S. Akdeniz 1094.

13. . arvensis L., Y, stony slopes, 23.04.2008, S. Akdeniz 1084.

14. Ceratocephalus falcatus (L.) Pers., Y, Akçaören, grassland, 855 m, 16.04.2009, S. Akdeniz 1213.

4. BERBERIDACEAE

15. Berberis crataegina DC., A, Mountain pass of Ayaş, stony slopes, 1060 m, 15.05.2009, S. Akdeniz 1276.

5. PAPAVERACEAE

16. Glaucium corniculatum (L.) Rud. subsp. refractum (Nab.) Cullen, K, step, 780 m, 08.07.2007, S. Akdeniz 1004., K, field edge, 800 m, 07.08.2007, S. Akdeniz 1045.

17. G. grandiflorum Boiss. \& Huet. var. torquatum Cullen, A, Mountain pass of Ayaş, 1060 m, 15.05.2009, S. Akdeniz 1281. Irano - Turanian. Endemic. EN.

18. Roemeria hybrida (L.) DC. subsp. hybrida, A, plowed land, 858 m, 28.05.2008, S. Akdeniz 1097.

19. Papaver rhoeas L., Y, roadside, 800 m, 31.05.2009, S. Akdeniz 1290.

20. P. commutatum Fisch. \& Mey. subsp. euxinum Kadereit, A, stony slopes, 960 m, 28.05.2008, S. Akdeniz 1098. Euxine. Endemic. EN.

21. P. syriacum Boiss. \& Blanche, A, Mountain pass of Ayaş, wasteland, 1060 m, 15.05.2009, S. Akdeniz 1249.

22. P. dubium L., A, cultivated land, 900 m, 25.06.2008, S. Akdeniz 1159., A, Mountain pass of Ayaş, wasteland, $1060 \mathrm{~m}, 15.05 .2009, \mathrm{~S}$. Akdeniz 1248.

23. Hypecoum procumbens L., Y, grassland, 850 m, 23.04.2008, S. Akdeniz 1071., A, field edge, $91 \mathrm{~m}$, 28.05.2008, S. Akdeniz 1096. Mediterranean.

24. H. imberbe Sibth. \& Sm., Y, Akçaören, grassland, 800 m, 16.04.2009, S. Akdeniz 1206.

25. H. pendulum L., A, Mountain pass of Ayaş, wasteland, 1060 m, 15.05.2009, S. Akdeniz 1250.

26. Fumaria vailantii Lois., A, Mountain pass of Ayaş, wasteland, 1060 m, 15.05.2009, S. Akdeniz 1280.

27. F. parviflora Lam., A, yola yakın wasteland, 920 m, 28.05.2008, S. Akdeniz 1099., A, cultivated land, 93 m, 25.06.2008, S. Akdeniz 1158.

6. BRASSICACEAE (CRUCIFERAE)

28. Brassica elongata Ehrh., Y, roadside, 855 m, 31.05.2009, S. Akdeniz 1333.

29. Sinapis arvensis L., A, wasteland, 960 m, 28.05.2008, S. Akdeniz 1102.

30. Hirschfeldia incana (L.) Lag. - Foss., Y, Akçaören, grassland, 800 m, 16.04.2009, S. Akdeniz 1201., Y, roadside, 855 m, 31.05.2009, S. Akdeniz 1331.

31.Diplotaxis tenuifolia (L.) DC., Y, roadside, 855 m, 31.05.2009, S. Akdeniz 1328.

32. Eruca sativa Miller, Y, roadside, 855 m, 31.05.2009, S. Akdeniz 1332. 
33. Raphanus raphanistrum L., K, cultivated areas, $710 \mathrm{~m}, 08.07 .2007$, S. Akdeniz 1023., A, step, $900 \mathrm{~m}$, 28.05.2008, S. Akdeniz 1103.

34. Conringia orientalis (L.) Andrz., K, step, 700 m, 08.07.2007, S. Akdeniz 1025.

35. C. perfoliata (C. A. Mey.) Busch, A, Mountain pass of Ayaş, stony slopes, $1060 \mathrm{~m}, 15.05 .2009$, S. Akdeniz 1224.

36. Lepidium perfoliatum L., Y, stony slopes, $780 \mathrm{~m}, 23.04 .2008$, S. Akdeniz 1069., Y, roadside, $855 \mathrm{~m}$, 31.05.2009, S. Akdeniz 1330.

37. Cardaria draba (L.) Desv. subsp. draba, Y, field edge, 790 m, 23.04.2008, S. Akdeniz 1062., Y, Akçaören, grassland, 16.04.2009, S. Akdeniz 1199., Y, roadside, 855 m, 31.05.2009, S. Akdeniz 1327.

38. Iberis taurica DC., A, Mountain pass of Ayaş, wasteland, 1060 m, 15.05.2009, S. Akdeniz 1228.

39. Aethionema capitatum Boiss. \& Bal., A, Mountain pass of Ayaş, wasteland, $1060 \mathrm{~m}, 15.05 .2009$, S. Akdeniz 1226. Endemic. NT.

40. Ae. armenum Boiss., A, Mountain pass of Ayaş, wasteland, 1060 m, 15.05.2009, S. Akdeniz 1225. Irano Turanian.

41. Thlaspi perfoliatum L., Y, IFN, grassland, 840 m, 16.04.2009, S. Akdeniz 1194., A, Mountain pass of Ayaş, wasteland, $1060 \mathrm{~m}, 15.05 .2009, \mathrm{~S}$. Akdeniz 1231.

42. T. violascens Boiss., A, Mountain pass of Ayaş, wasteland, 1060 m, 15.05.2009, S. Akdeniz 1230 Endemic. LC.

43. Capsella bursa-pastoris (1.) Medik., Y, field edge, 850 m, 23.04.2008, S. Akdeniz 1061., Y, Akçaören, grassland, 860 m, 16.04.2009, S. Akdeniz 1197., Y, roadside, 855 m, 31.05.2009, S. Akdeniz 1326.

44. Boreava orientalis Jaub. \& Spach, Y, roadside, 23.04.2008, S. Akdeniz 1076., A, Mountain pass of Ayaş, wasteland, 1060 m, 15.05.2009, S. Akdeniz 1227.

45. Myagrum perfoliatum L., A, Mountain pass of Ayaş, wasteland, 1060 m, 15.05.2009, S. Akdeniz 1235.

46. Alyssum linifolium Steph ex Wild. var. linifolium, Y, IFN, grassland, 830 m, 16.04.2009, S. Akdeniz 1203., A, Mountain pass of Ayaş, wasteland, 1060 m, 15.05.2009, S. Akdeniz 1245.

47. A. blepharocarpum Dudley \& Hub. - Mor., Y, Akçören, wasteland, 800 m, 16.04.2009, S. Akdeniz 1205a., Y, IFN, grassland, $810 \mathrm{~m}, 16.04 .2009, \mathrm{~S}$. Akdeniz 1205b.

A, Mountain pass of Ayaş, wasteland, 1060 m, 15.05.2009, S. Akdeniz 1240. Irano - Turanian. Endemic. NT.

48. A. alyssoides (L.) L., Y, destroyed lands, 855 m, 23.04.2008, S. Akdeniz 1078., Y, Akçören, wasteland, 800 m, 16.04.2009, S. Akdeniz 1204.

49. A. desertorum Stapf. var. desertorum, A, wasteland, 950 m, 28.05.2008, S. Akdeniz 1101., A, Mountain pass of Ayaş, wasteland, 1060 m, 15.05.2009, S. Akdeniz 1239.

50. A. minus (L.) Rothm. var. micranthum (Mey.) Dudley, A, Mountain pass of Ayaş, wasteland, $1060 \mathrm{~m}$, 15.05.2009, S. Akdeniz 1242.

51. A. stapfii Vierh., A, Mountain pass of Ayaş, wasteland, 1060 m, 15.05.2009, S. Akdeniz 1238. Irano Turanian.

52. A. strigosum Banks \& Sol. subsp. cedrorum (Schott \& Kotschy) Dudley, A, Mountain pass of Ayaş, wasteland, 1060 m, 15.05.2009, S. Akdeniz 1243.

53.A. xanthocarpum Boiss., A, Mountain pass of Ayaş, wasteland, 1060 m, 15.05.2009, S. Akdeniz 1237.

54. A. hirsutum Bieb.var. caespitosum Dudley, A, Mountain pass of Ayaş, wasteland, 1060 m, 15.05.2009, S. Akdeniz 1244. Irano - Turanian. Endemic. NT.

55.A. paphlagonicum (Hausskn.) Dudley, A, Mountain pass of Ayaş, wasteland, $1060 \mathrm{~m}, 15.05 .2009$, S. Akdeniz 1236. Irano - Turanian. Endemic. LC.

56. A. tortuosum Willd., A, Mountain pass of Ayaş, wasteland, 1060 m, 15.05.2009, S. Akdeniz 1241. Euro Siberian.

57. Erophila verna (L.) Chevall subsp. verna, Y, IFN, sloping terrain, 855 m, 16.04.2009, S. Akdeniz 1202.

58. Arabis nova Vill., A, wasteland, 960 m, 28.05.2008, S. Akdeniz 1100., A, Mountain pass of Ayaş, wasteland, $1060 \mathrm{~m}, 15.05 .2009$, S. Akdeniz 1233.

59. Matthiola longipetala (Vent.) DC. subsp. bicornis (Sibth. \& Smith) P.W. Ball, A, Mountain pass of Ayaş, wasteland, $1060 \mathrm{~m}, 15.05 .2009$, S. Akdeniz 1232.

60. Chorispora syriaca Boiss., Y, grassland, 800 m, 23.04.2008, S. Akdeniz 1075., Y, Akçaören, cultivated land, 16.04.2009, S. Akdeniz 1200. Irano - Turanian.

61. Hesperis balansae Fourn. subsp. balansae Fourn., A, Mountain pass of Ayaş, wasteland, 1060 m, 15.05.2009, S. Akdeniz 1234., East Mediterranean. Endemic. EN.

62. Erysimum thyrsoideum Boiss. subsp. ponticum (Hausskn. \& Bornm. ) Cullen, A, Mountain pass of Ayaş, wasteland, 1060 m, 15.05.2009, S. Akdeniz 1246. Endemic. LC.

63. E. smyrnaeum Boiss. \& Bal., A, Mountain pass of Ayaş, wasteland, 1060 m, 15.05.2009, S. Akdeniz 1247.

64. E. repandum L., Y, wasteland, 880 m, 23.04.2008, S. Akdeniz 1068. 
65. Sisymbrium altissimum L., A, cultivated land, 970 m, 25.06.2008, S. Akdeniz 1157., Y, roadside, 855 m, 31.05.2009, S. Akdeniz 1329.

66. Descurainia sophia (L.) Webb. ex Prantl, Y, wasteland, 855 m, 23.04.2008, S. Akdeniz 1070., A, abandoned lands, 960 m, 28.05.2008, S. Akdeniz 1104., Y, Akçaören, grassland, 800 m, 16.04.2009, S. Akdeniz 1195.

67. Camelina laxa C. A. Mey., A, step, 960 m, 28.05.2008, S. Akdeniz 1105.

68. C. rumelica Vel., A, Mountain pass of Ayaş, wasteland, 1060 m, 15.05.2009, S. Akdeniz 1229.

69. C. hispida Boiss. var. hispida, A, wasteland, 960 m, 28.05.2008, S. Akdeniz 1106.

7. RESEDACEAE

70. Reseda lutea L. var. lutea, K, dere yatağ1, 780 m, 07.08.2007, S. Akdeniz 1047.

71. R. lutea L. var. nutans Boiss., A, field, 1000 m, 28.05.2008, S. Akdeniz 1122.

8. CISTACEAE

72. Helianthemum nummularium (L.) Miller subsp. ovatum (Viv.) Schinz \& Thellung, A, Mountain pass of Ayaş, wasteland, 1060 m, 15.05.2009, S. Akdeniz 1263.

\section{VIOLACEAE}

73. Viola occulta Lehm., Y, IFN, grassland, $800 \mathrm{~m}, 16.04 .2009$, S. Akdeniz 1211., A, Mountain pass of Ayaş, wasteland, $1060 \mathrm{~m}, 15.05 .2009$, S. Akdeniz 1264.

74. V. parvula Tineo., Y, grassland, 780 m, 23.04.2008, S. Akdeniz 1072.

75. V. kitaibeliana Roem. \& Schult., A, Mountain pass of Ayaş, wasteland, 1060 m, 15.05.2009, S. Akdeniz 1265.

10. CARYOPHYLLACEAE

76. Arenaria serpyllifolia L., A, Mountain pass of Ayaş, wasteland, 1060 m, 15.05.2009, S. Akdeniz 1312.

77. Cerastium chlorifolium Fisch. \& Mey., A, field, 960 m, 28.05.2008, S. Akdeniz 1310.

78. C. perfoliatum L., A, Mountain pass of Ayaş, wasteland, 1060 m, 15.05.2009, S. Akdeniz 1311.

79. Holosteum marginatum C. A. Mey., A, Mountain pass of Ayaş, wasteland, 1060 m, 15.05.2009, S. Akdeniz 1309.

80. H. umbellatum L. var. umbellatum, A, grassland, 990 m, 23.04.2008, S. Akdeniz 1092., A, Mountain pass of Ayaş, wasteland, $1060 \mathrm{~m}, 15.05 .2009$, S. Akdeniz 1308.

81. H. umbellatum L. var. glutinosum (Bieb.) Gay, Y, Akçaören, grassland, 780 m, 16.04.2009, S. Akdeniz 1306., A, Mountain pass of Ayaş, wasteland, 1060 m, 15.05.2009, S. Akdeniz 1307.

82. Silene supina Bieb. subsp. pruniosa (Boiss.) Chowdh., A, Mountain pass of Ayaş, wasteland, $1060 \mathrm{~m}$, 15.05.2009, S. Akdeniz 1313.

83. S. vulgaris (Moench) Garcke var. vulgaris, A, cemetery, 970 m, 28.05.2008, S. Akdeniz 1123.

84.S. dichotoma Ehrh. subsp. dichotoma, A, wasteland, 990 m, 28.05.2009, S. Akdeniz 1314.

11. GUTTIFERAE

85. Hypericum perforatum L., A, field, 960 m, 25.06.2008, S. Akdeniz 1162.

12. GERANIACEAE

86. Geranium tuberosum L. subsp. tuberosum, Y, stony slopes, 780 m, 23.04.2008, S. Akdeniz 1060., A, Mountain pass of Ayaş, wasteland, $1060 \mathrm{~m}, 15.05 .2009$, S. Akdeniz 1270.

87. Erodium absinthoides Willd. subsp. absinthoides, A, Mountain pass of Ayaş, wasteland, 1060 m, 15.05.2009, S. Akdeniz 1269., Irano - Turanian. Endemic. LC.

88. E. cicutarium (L.) L'Hérit subsp. cicutarium, Y, step, 800 m, 23.04.2008, S. Akdeniz 1065., A, wasteland, 980 m, 28.05.2008, S. Akdeniz 1120., Y, Akçaören, grassland, 800 m, 16.04.2009, S. Akdeniz 1209.

89. E. acaule (L.) Becherer \& Thell, Y, grassland, 855 m, 16.04.2009, S. Akdeniz 1210. Mediterranean.

13. RHAMNACEAE

90. Paliurus spina-christi Miller, A, forestland, 1020 m, 28.05.2008, S. Akdeniz 1119.

14. FABACEAE (LEGUMINOSAE)

91. Genista sessilifolia DC., A, Mountain pass of Ayaş, eroded land, 1060 m, 15.05.2009, S. Akdeniz 1285. Irano Turanian.

92. Colutea cilicica Boiss. \& Bal, K, dere yatağ 1,780 m, 07.08.2007, S. Akdeniz 1046.

93. Astragalus microcephalus Willd., Y, Akçaören, eroded land, 790 m, 16.04.2009, S. Akdeniz 1212. Irano Turanian.

94. A. ponticus Pall., A, step, 950 m, 25.06.2008, S. Akdeniz 1166.

95. A. lycius Boiss., A, Mountain pass of Ayaş, wasteland, 1060 m, 15.05.2009, S. Akdeniz 1288. Endemic. LC.

96. A. xylobasis Freyn. \& Bornm. var. angustus (Freyn. \& Sint.) Freyn. \& Bornm., A, wasteland, $980 \mathrm{~m}$, 25.06.2008, S. Akdeniz 1319. Irano - Turanian. Endemic. LC.

97. A. hirsutus Vahl., A, Mountain pass of Ayaş, wasteland, 1060 m, 15.05.2009, S. Akdeniz 1355. Endemic. LC. 98. A. humillimus Freyn. \& Sint., A, Mountain pass of Ayaş, wasteland, 1060 m, 15.05.2009, S. Akdeniz 1318. Euxine. Endemic. LC. 
99. A. sigmoideus Bunge., A, Mountain pass of Ayaş, wasteland, 1060 m, 15.05.2009, S. Akdeniz 1346. Endemic. LC.

100. Vicia sativa L. subsp. sativa, A, Mountain pass of Ayaş, wasteland, 1060 m, 15.05.2009, S. Akdeniz 1320.

101. Lathyrus digitatus (Bieb.) Fiori., A, field edge, 970 m, 28.05.2008, S. Akdeniz 1154., East Mediterranean.

102. Ononis spinosa L. subsp. leiosperma (Boiss.) Sirj., A, eroded land, 980 m, 25.06.2008, S. Akdeniz 1168.

103. Trifolium resupinatum L. var. microcephalum Zoh., Y, roadside, 855 m, 31.05.2009, S. Akdeniz 1356.

104. Melilotus officinalis (L.) Desr.

A, wasteland, 980 m, 28.05.2008, S. Akdeniz 1152., Y, roadside, 855 m, 31.05.2009, S. Akdeniz 1325.

105. Trigonella rostrata (Boiss. \& Bal.) Boiss., A, Mountain pass of Ayaş, wasteland, $1060 \mathrm{~m}, 15.05 .2009$, S. Akdeniz 1321. Irano - Turanian. Endemic. NT.

106. T. spruneriana Boiss. var. spruneriana, A, Mountain pass of Ayaş, wasteland, $1060 \mathrm{~m}, 15.05 .2009$, S. Akdeniz 1317. Irano - Turanian.

107. T. aurantiaca Boiss., Y, roadside, $855 \mathrm{~m}, 31.05 .2009$, S. Akdeniz 1316. Irano - Turanian.

108. T. fischeriana Ser., A, cultivated area, $950 \mathrm{~m}, 28.05 .2008$, S. Akdeniz 1153. Irano - Turanian

109. T. monantha C. A. Meyer subsp. monantha, A, Mountain pass of Ayaş, wasteland, $1060 \mathrm{~m}, 15.05 .2009, \mathrm{~S}$. Akdeniz 1286. Irano - Turanian.

110. Medicago sativa L. subsp. sativa, K, wasteland, 780 m, 08.07.2009, S. Akdeniz 1012, 07.08.2007, S. Akdeniz 1041., A, stony slopes, 980 m, 28.05.2008, S. Akdeniz 1155, 25.06.2008, S. Akdeniz 1169.

111. M. minima (L.) Bart. var. minima, A, Mountain pass of Ayaş, wasteland, $1060 \mathrm{~m}, 15.05 .2009$, S. Akdeniz 1287.

112. M. truncatula Gaertn. var. longiaculeata, A, Mountain pass of Ayaş, wasteland, $1060 \mathrm{~m}, 15.05 .2009$, S. Akdeniz 1357. Mediterranean.

113. M. rigidula (L.) All. var. rigidula, A, Mountain pass of Ayaş, wasteland, $1060 \mathrm{~m}, 15.05 .2009$, S. Akdeniz 1315.

114. Coronilla varia L. subsp. varia, A, cultivated areas, 980 m, 25.06.2008, S. Akdeniz 1167.

115. Hedysarum varium Willd., K, step, 780 m, 08.07.2007, S. Akdeniz 1001. Irano - Turanian.

116. Onobrychis armena Boiss. \& Huet., A, field edge, 980 m, 25.06.2008, S. Akdeniz 1170. Endemic. LC.

117. O. oxyodontha Boiss., A, cemetery, 970 m, 28.05.2008, S. Akdeniz 1156.

118. O. hypargyrea Boiss., A, field, 990 m, 25.06.2008, S. Akdeniz 1171.

15. ROSACEAE

119. Prunus divaricata Ledeb. subsp. divaricata, A, stony slopes, 980 m, 28.05.2008, S. Akdeniz 1126.

120. Cerasus mahaleb (L.) Miller var. mahaleb, Y, wasteland, 850 m, 23.04.2008, S. Akdeniz 1067.

121. Potentilla recta L., A, stony slopes, 980 m, 28.05.2008, S. Akdeniz 1125.

122. Rosa canina L., K, destroyed lands, 780 m, 07.08.2007, S. Akdeniz 1048., A, stony slopes, 980 m, 28.05.2008, S. Akdeniz 1124.

123. Crataegus orientalis Pallas ex Bieb. var. orientalis, Y, rocky slopes, 810 m, 20.10.2007, S. Akdeniz 1053.

124. Pyrus eleagnifolia Pallas. subsp. eleagnifolia, A, stony slopes, 980 m, 28.05.2008, S. Akdeniz 1127.

16. APIACEAE (UMBELLIFERAE)

125. Echinophora tenuifolia subsp. sibtropiana (Guss.) Tutin, K, destroyed lands, 705 m, 08.07.2007, S. Akdeniz 1030 .

126. Coriandrum sativum L., A, stony slopes, 980 m, 28.05.2008, S. Akdeniz 1031.

127. Fuernrohria setifolia C. Koch., A, stony slopes, 980 m, 28.05.2008, S. Akdeniz 1129.

128. Bifora radians Bieb., A, stony slopes, 980 m, 28.05.2008, S. Akdeniz 1128., Y, roadside, 855 m, 31.05.2009, S. Akdeniz 1335.

129. Ferula rigidula DC., K, wasteland, $780 \mathrm{mi}, 08.07 .2007$, S. Akdeniz 1026. Irano - Turanian.

130. Malabaila secacul Banks \& Sol., A, stony slopes, 980 m, 28.05.2008, S. Akdeniz 1133.

131. Astradaucus orientalis (L.) Drude, K, dere yatağ, 770 m, 07.08.2007, S. Akdeniz 1034.

132. Turgenia latifolia (L.) Hoffm., A, stony slopes, 980 m, 28.05.2008, S. Akdeniz 1132.

133. Daucus carota L., K, step, 780 m, 08.07.2007, S. Akdeniz 1022., A, stony slopes, 980 m, 28.05.2008, S. Akdeniz 1130.

\section{VALERIANACEAE}

134.Valerianella coronata (L.) DC., A, stony slopes, 980 m, 28.05.2008, S. Akdeniz 1109.

135. Valerianella vesicaria (L.) Moench, A, Mountain pass of Ayaş, wasteland, $1060 \mathrm{~m}, 15.05 .2009$, S. Akdeniz 1272.

18. DIPSACACEAE

136. Cephalaria syriaca (L.) Schrader, K, step, 780 m, 08.07.2007, S. Akdeniz 1008.

137. Scabiosa argentea L., A, stony slopes, 980 m, 25.06.2008, S. Akdeniz 1172. 
138. S. rotata Bieb., K, step, 780 m, 08.07.2007, S. Akdeniz 1006., A, stony slopes, 980 m, 25.06.2008, S. Akdeniz 1173., A, Mountain pass of Ayaş, wasteland, 1060 m, 15.05.2009, S. Akdeniz 1268. Irano - Turanian.

\section{ASTERACEAE (COMPOSITAE)}

139. Inula montbretiana DC., A, stony slopes, 980 m, 25.06.2008, S. Akdeniz 1190. Irano - Turanian.

140. Helichrysum noeanum Zahn., K, step, 780 m, 08.07.2007, S. Akdeniz 1014. Irano - Turanian. Endemic. LC.

141. Conyza canadensis (L.) Cronquist, K, step, 780 m, 07.08.2007, S. Akdeniz 1039.

142. Senecio vernalis Waldst. \& Kit., A, stony slopes, 980 m, 28.05.2008, S. Akdeniz 1142., A, roadside, 950 m, 25.06.2008, S. Akdeniz 1184., Y, Akçaören, wasteland, 850 m, 16.04.2009, S. Akdeniz 1220., A, Mountain pass of Ayaş, wasteland, $1060 \mathrm{~m}, 15.05 .2009$, S. Akdeniz 1283.

143. S. viscosus L., Y, wasteland, 850 m, 23.04.2008, S. Akdeniz 1086.

144. Anthemis cretica L. subsp. pontica (Willd.) Grierson, A, Mountain pass of Ayaş, wasteland, $1060 \mathrm{~m}$, 15.05.2009, S. Akdeniz 1256.

145. A. cretica L. subsp. albida (Boiss.) Grierson, A, Mountain pass of Ayaş, wasteland, $1060 \mathrm{~m}, 15.05 .2009, \mathrm{~S}$. Akdeniz 1255.

146. A. cretica L. subsp. tenuiloba (DC.) Grierson, A, stony slopes, 980 m, 28.05.2008, S. Akdeniz 1138.

147. A. tinctoria L. var. pallida DC., A, roadside, $950 \mathrm{~m}, 28.05 .2008$, S. Akdeniz 1137.

148. A. austriaca Jacq., Y, roadside, 850 m, 31.05.2009, S. Akdeniz 1289.

149. Achillea wilhelmsii C. Koch., A, stony slopes, 980 m, 28.05.2008, S. Akdeniz 1145. Irano - Turanian.

150. A. phrygia Boiss. \& Bal., Y, wasteland, 850 m, 23.04.2008, S. Akdeniz 1085. Irano - Turanian. Endemic. LC.

151. A. millefolium L. subsp. millefolium, A, stony slopes, 980 m, 25.06.2008, S. Akdeniz 1186. Euro - Siberian.

152. A. bieberstenii Afan., A, roadside, $950 \mathrm{~m}, 25.06 .2008$, S. Akdeniz 1359., Irano - Turanian.

153. Tanacetum armenum (DC.) Schultz Bip., Y, Akçaören, grassland, 855 m, 16.04.2009, S. Akdeniz 1221.

154. Artemisia austriaca Jacq., K, Orhaniye, step, 780 m, 07.08.2007, S. Akdeniz 1033.

155. Causinia iconica Hub. - Mor., K, step, 800 m, 08.07.2007, S. Akdeniz 1028. Irano - Turanian. Endemic. NT.

156. Onopordum acanthium L., K, step, 780 m, 08.07.2007, S. Akdeniz 1018.

157. Cirsium vulgare (Savi) Ten., A, Mountain pass of Ayaş, wasteland, 1060 m, 15.05.2009, S. Akdeniz 1282.

158. C. leucopsis DC., Y, Mülkköy, field, 850 m, 20.10.2007, S. Akdeniz 1056., East Mediterranean. Endemic. LC.

159. C. arvense (L.) Scop. subsp. vestitum (Wimmer \& Grab.) Petrak, Y, roadside, 855 m, 31.05.2009, S. Akdeniz 1323.

160. Carduus nutans L. subsp. nutans sensu lato, A, roadside, 950 m, 28.05.2008, S. Akdeniz 1135.

161. C. pycnocephalus L. subsp. albidus (Bieb. ) Kazmi, A, field, 950 m, 28.05.2008, S. Akdeniz 1136.

162. Acroptilon repens (L.) DC. K, step, 780 m, 08.07.2007, S. Akdeniz 1015. Irano - Turanian.

163. Centaurea virgata Lam., K, step, 780 m, 08.07.2007, S. Akdeniz 1020, 07.08.2007, S. Akdeniz 1036., Y, wasteland, 850 m, 20.10.2007, S. Akdeniz 1058., A, step, 980 m, 25.06.2008, S. Akdeniz 1187. Irano - Turanian.

164. C. paphlagonica (Bornm.) Wagenitz, K, step, 780 m, 07.08.2007, S. Akdeniz 1038. Endemic. NT.

165. C. solstitialis L. subsp. solstitialis, K, step, 780 m, 08.07.2007, S. Akdeniz 1016.

166. C. urvillei DC. subsp. urvillei , A, stony slopes, 980 m,25.06.2008, S. Akdeniz 1188., East Mediterranean.

167. C. triumfettii All., A, Mountain pass of Ayaş, wasteland, 1060 m, 15.05.2009, S. Akdeniz 1257.

168. C. depressa Bieb., K, step, 780 m, 08.07.2007, S. Akdeniz 1017., A, stony slopes, 980 m, 28.05.2008, S. Akdeniz 1144, 25.06.2008, S. Akdeniz 1189., Y, roadside, 855 m, 31.05.2009, S. Akdeniz 1322.

169. Xeranthemum annuum L., K, step, 780 m, 08.07.2007, S. Akdeniz 1019, 07.08.2007, S. Akdeniz 1035.

170. Echinops ritro L., K, step, 780 m, 07.08.2007, S. Akdeniz 1037., Y, wasteland, 850 m, 20.10.2007, S. Akdeniz 1057.

171. E. microcephala Sm., Y, roadside, 855 m, 08.07.2007, S. Akdeniz 1029. Mediterranean.

172. Cichorium intybus L., Y, wasteland, 850 m, 20.10.2007, S. Akdeniz 1059.

173. Scorzonera cana (C. A. Meyer) Hoffm. var. cana, A, Mountain pass of Ayaş, wasteland, 1060 m, 15.05.2009, S. Akdeniz 1257.

174. Tragopogon dubius Scop., Y, roadside, 855 m, 28.05.2008, S. Akdeniz 1143, 25.06.2008, S. Akdeniz 1185.

175. Taraxacum serotinum (Waldst \& Kit) Poiret, Y, Fethiye, grassland, 860 m, 29.10.2008, S. Akdeniz 1191.

176. T. phaleratum G. Hagl.ex Rech.

Y, IFN, wasteland, 16.04.2009, S. Akdeniz 1192, 16.04.2009, S. Akdeniz 1193.

177. Chondrilla juncea L. var. juncea, K, step, 780 m, 07.08.2007, S. Akdeniz 1040.

178. Crepis macropus Boiss. \& Heldr., Y, wasteland, 850 m, 20.10.2007, S. Akdeniz 1055. Endemic. LC.

179. C. pulchra L. subsp. pulchra, A, roadside, 950 m, 28.05.2008, S. Akdeniz 1139. 
180. C. alpina L., A, stony slopes, 980 m, 28.05.2008, S. Akdeniz 1141.

181. C. foetida L. subsp. rhoeadifolia (Bieb.) Čelak., K, step, 780 m, 08.07.2007, S. Akdeniz 1013, 1021.

182. C. sancta (L.) Babc., Y, wasteland, 850 m, 23.04.2008, S. Akdeniz 1087., A, stony slopes, 980 m, 28.05.2008, S. Akdeniz 1140., Y, IFN, Akçaören, 850 m, 16.04.2009, S. Akdeniz 1218, 16.04.2009, S. Akdeniz 1219., A, Mountain pass of Ayaş, wasteland, 1060 m, 15.05.2009, S. Akdeniz 1284.

183. C. setosa Hall., K, step, 780 m, 08.07.2009, S. Akdeniz 1027.

\section{PRIMULACEAE}

184. Anagallis arvensis L. var. caerulea (L.) Gouan, A, stony slopes, 980 m, 28.05.2008, S. Akdeniz 1110

21. APOCYNACEAE

185. Vinca herbacea Waldst. \& Kit., A, Mountain pass of Ayaş, wasteland, 1060 m, 15.05.2009, S. Akdeniz 1271.

22. CONVOLVULACEAE

186. Convolvulus arvensis L., K, roadside, 780 m, 08.07.2007, S. Akdeniz 1005, 07.08.2007, S. Akdeniz 1044., A, roadside, 950 m, 28.05.2008, S. Akdeniz 1116, 25.06.2008, S. Akdeniz 1160., Y, roadside, 855 m, 31.05.2009, S. Akdeniz 1334.

\section{CUSCUTACEAE}

187. Cuscuta Europaea L., A,stony slopes, 980 m, 25.06.2008, S. Akdeniz 1165.

24. BORAGINACEAE

188. Myosotis ramosissima Rochel ex Schultes subsp. ramosissima, A, Mountain pass of Ayaş, wasteland, $1060 \mathrm{~m}$, 15.05.2009, S. Akdeniz 1275.

189. Neotestema apulum (L.) Johnston, Y, wasteland, 850 m, 23.04.2008, S. Akdeniz 1084. Mediterranean.

190. Moltkia coerulea (Willd.) Lehm., Y, wasteland, $850 \mathrm{~m}, 20.10 .2007$, S. Akdeniz 1052., A, Mountain pass of Ayaş, wasteland, 1060 m, 15.05.2009, S. Akdeniz 1274. Irano - Turanian.

191. Onosma tauricum Pallas ex Willd. var. brevifolium DC., A, Mountain pass of Ayaş, wasteland, $1060 \mathrm{~m}$, 15.05.2009, S. Akdeniz 1273. Endemic. LC.

192. Cerinthe minor L. subsp. auriculata (Ten.) Domac, A, stony slopes, 980 m, 28.05.2008, S. Akdeniz 1111, 25.06.2008, S. Akdeniz 1161.

193. Anchusa leptophylla Roemer \& Schuter subsp. leptophylla, Y, wasteland, 850 m, 23.04.2008, S. Akdeniz 1082., A, stony slopes, 980 m, 28.05.2008, S. Akdeniz 1114.

194. A. undulata L. subsp. hybrida (Ten.) Countinho,A,roadside,950 m, 28.05.2008, S. Akdeniz 1115., Mediterranean.

195. A. azurea Miller var. azurea, $\mathrm{Y}$, wasteland, $850 \mathrm{~m}, 23.04 .2008$, S. Akdeniz 1083., A, roadside, $950 \mathrm{~m}$, 28.05.2008, S. Akdeniz 1112.

196. A. pusilla Guşul, Y, Roadside, 855 m, 23.04.2008, S. Akdeniz 1081., A, roadside, 950 m, 28.05.2008, S. Akdeniz 1113., Y, Akçaören, grassland, 850 m, 16.04.2009, S. Akdeniz 1208

\section{SOLANACEAE}

197. Hyosyamus niger L., A, stony slopes, 980 m, 28.05.2008, S. Akdeniz 1118.

26. SCROPHULARIACEAE

198. Verbascum lasianthum Boiss. ex Bentham, A, roadside, 950 m, 25.06.2008, S. Akdeniz 1182.

199. V.cheIranthifolium Boiss. var. asperulum (Boiss.)Murb., K, step, 780 m, 08.07.2007, S. Akdeniz 1031. Endemic. LC.

200. Linaria corifolia Desf., A, Mountain pass of Ayaş, wasteland, 1060 m, 15.05.2009, S. Akdeniz 1259. Irano Turanian. Endemic. LC.

201. L. simplex (Willd.) DC., A, Mountain pass of Ayaş, wasteland, 1060 m,15.05.2009, S. Akdeniz 1258. Mediterranean.

202. Digitalis lamarckii Ivan., A, stony slopes, 980 m, 25.06.2008, S. Akdeniz 1183., Irano - Turanian. Endemic. LC.

203. Veronica arvensis L., A, Mountain pass of Ayaş, wasteland, 1060 m, 15.05.2009, S. Akdeniz 1260. Euro Siberian.

204. V. triphillos L., Y, wasteland, 850 m, 23.04.2008, S. Akdeniz 1079, Akçaören, 16.04.2009, S. Akdeniz 1214., A, stony slopes, $980 \mathrm{~m}, 28.05 .2008$, S. Akdeniz 1134.

205. V. persica Pioret., Y, wasteland, 850 m, 23.04.2008, S. Akdeniz 1089, Akçaören, 16.04.2009, S. Akdeniz 1215. 206. V. triloba (Opiz.) Kerner, Y, Akçaören, wasteland, 850 m, 16.04.2009, S. Akdeniz 1216.

207. V. hederifolia L., Y, wasteland, 850 m, 23.04.2008, S. Akdeniz 1080, Akçaören, 16.04.2009, S. Akdeniz 1217. 208. V. multifida L., Y, wasteland, 850 m, 23.04.2008, S. Akdeniz 1063., A, Mountain pass of Ayaş, wasteland, 1060 m, 15.05.2009, S. Akdeniz 1261. Irano - Turanian. Endemic. LC.

209. Bungea trifida (Vahl) C. A. Meyer, A, Mountain pass of Ayaş, wasteland, $1060 \mathrm{~m}, 15.05 .2009$, S. Akdeniz 1262. Irano - Turanian.

27. OROBANCHACEAE 
210. Orobanche anatolica Boiss. \& Reuter, A, Mountain pass of Ayaş, wasteland, 1060 m, 15.05.2009, S. Akdeniz 1358.

28. GLOBULARIACEAE

211. Globularia trichosantha Fisch. \& Mey., A, forestland, $980 \mathrm{~m}, 28.05 .2008$, S. Akdeniz 1121., Y, IFN, wasteland, 850 m, 16.04.2009, S. Akdeniz 1207., A, Mountain pass of Ayaş, wasteland, 1060 m, 15.05.2009, S. Akdeniz 1277.

\section{LAMIACEAE (LABIATAE)}

212. Ajuga chamaepitys (L.) Schreber subsp. chia (Schreber) Arcangeli var. chia, K, step, 780 m, 08.07.2007, S. Akdeniz 1010., A, roadside, 950 m, 28.05.2008, S. Akdeniz 1148.

213. Teucrium chamaedrys L. subsp. chamaedrys L., A, stony slopes, 980 m, 25.06.2008, S. Akdeniz 1176. EuroSiberian.

214. T. polium L., K, step, 780 m, 08.07.2007, S. Akdeniz 1032., A, stony slopes, 980 m, 25.06.2008, S. Akdeniz 1177.

215. Scutellaria orientalis L. subsp. pinnatifida Edmondson, A, stony slopes, 980 m, 28.05.2008, S. Akdeniz 1147. 216. Phlomis pungens Willd. var. pungens, K, step, 780 m, 08.07.2007, 25.06.2008, S. Akdeniz 1179.

217. P. armeniaca Willd., A, stony slopes, 980 m, 25.06.2008, S. Akdeniz 1178. Irano - Turanian. Endemic. LC. 218. Lamium garganicum L. subsp. reniforme (Montbret \& Aucher ex Bentham) R. Mill, A, stony slopes, $980 \mathrm{~m}$, 28.05.2008, S. Akdeniz 1150.

219. L. amplexicaule L., Y, wasteland, $850 \mathrm{~m}, 23.04 .2008$, S. Akdeniz 1088, Akçaören 16.04.2009, S. Akdeniz 1222, IFN, 16.04.2009, S. Akdeniz 1223., A, Mountain pass of Ayaş, wasteland, 1060 m, 15.05.2009, S. Akdeniz 1253. Irano - Turanian.

220. Wiedemannia orientalis Fisch. \& Mey., Y, roadside, 855 m, 23.04.2008, S. Akdeniz 1091., A, stony slopes, 980 m, 28.05.2008, S. Akdeniz 1146, Mountain pass of Ayaş, wasteland, 1060 m, 15.05.2009, S. Akdeniz 1254. Irano - Turanian. Endemic. LC.

221. Stachys cretica L. subsp. anatolica Rech., A, stony slopes, 980 m, 25.06.2008, S. Akdeniz 1175. Irano Turanian. Endemic. LC.

222. S. annua (L.) L. subsp. annua var. lycaonica Bhattacharjee, A, stony slopes, 980 m, 25.06.2008, S. Akdeniz 1174. Irano - Turanian.

223. Thymus sipyleus Boiss. subsp. rosulans (Borbàs) Jalas, A, Mountain pass of Ayaş, wasteland, $1060 \mathrm{~m}$, 15.05.2009, S. Akdeniz 1251.

224. T. leucostomus Hausskn. \& Velen. var. leucostomus, A, stony slopes, 980 m, 25.06.2008, S. Akdeniz 1181.

225. T. longicaulis C. Presl subsp. longicaulis var. subisophyllus (Barbàs) Jalas, A, stony slopes, $980 \mathrm{~m}$, 28.05.2008, S. Akdeniz 1149.

226. Salvia cryptantha Montbret \& Aucher, A, Mountain pass of Ayaş, wasteland, 1060 m, 15.05.2009, S. Akdeniz 1252. Irano - Turanian. Endemic. LC.

227. S. syriaca L., A, stony slopes, $980 \mathrm{~m}, 28.05 .2008$, S. Akdeniz 1151. Irano - Turanian.

228. S. virgata Jacq., A, stony slopes, 980 m, 25.06.2008, S. Akdeniz 1180. Irano - Turanian.

30. ELAEGNACEAE

229. Elaeagnus angustifolia L., K, step, 780 m, 07.08.2007, S. Akdeniz 1050., Y, wasteland, 850 m, 23.04.2008, S. Akdeniz 1073.

\section{EUPHORBIACEAE}

230. Euphorbia falcata L. subsp. falcata var. falcata, A, stony slopes, 980 m, 28.05.2008, S. Akdeniz 1107.

231. E. macroclada Boiss., K, step, 780 m, 08.07.2007, S. Akdeniz 1011. Irano - Turanian.

232. E. virgata Waldst. \& Kit., A, stony slopes, 980 m, 28.05.2008, S. Akdeniz 1108.

32. JUGLANDACEAE

233. Juglans regia L., Y, wasteland, 850 m, 23.04.2008, S. Akdeniz 1066.

\section{FAGACEAE}

234. Quercus pubescens Willd., A, stony slopes, 980 m, 28.05.2008, S. Akdeniz 1117, Mountain pass of Ayaş, wasteland, $1060 \mathrm{~m}, 15.05 .2009$, S. Akdeniz 1278.

34. RUBIACEAE

235. Asperula stricta Boiss. subsp. latibracteata (Boiss.) Ehrend, Y, Akçaören, wasteland, 857 m, 20.10.2007, S. Akdeniz 1051. Irano - Turanian. Endemic. LC.

236. A. arvensis L., A, Mountain pass of Ayaş, wasteland, 1060 m, 15.05.2009, S. Akdeniz 1267. Mediterranean. 237. Galium verum L. subsp. verum, A, stony slopes, 980 m, 25.06.2008, S. Akdeniz 1163. Euro - Siberian.

238. G. incanum Sm. subsp. elatius (Boiss.) Ehrend, A, stony slopes, 980 m, 25.06.2008, S. Akdeniz 1164. Irano Turanian.

239. Cruciata taurica (Pallas ex Willd.) Ehrend, A, Mountain pass of Ayaş, wasteland, 1060 m, 15.05.2009, S. Akdeniz 1266. Irano - Turanian. 
240. Rubia tinctorum L., K, step, 780 m, 07.08.2007, S. Akdeniz 1042. Irano - Turanian.

\section{LILIACEAE}

\section{MONOCOTYLEDONAE}

241. Allium paniculatum L. subsp. paniculatum, K, step, 780 m, 07.08.2007, S. Akdeniz 1338. Mediterranean. 242. A. scorodoprasum L. subsp. rotundum (L.) Stearn, A, stony slopes, 980 m, 25.06.2008, S. Akdeniz 1339. Mediterranean.

243. Ornithogalum sphareocarpum Kerner, Y, wasteland, 850 m, 23.04.2008, S. Akdeniz 1360.

244. O. narbonense L., A, stony slopes, 980 m, 28.05.2008, S. Akdeniz 1342. Mediterranean.

245. O. umbellatum L., Y, wasteland, 850 m, 23.04.2008, S. Akdeniz 1341.

246. O. orthophyllum Ten., A, Mountain pass of Ayaş, wasteland, 1060 m, 15.05.2009, S. Akdeniz 1340.

247. Muscari armeniacum Leichtlin ex Baker, A, Mountain pass of Ayaş, wasteland, $1060 \mathrm{~m}, 15.05 .2009$, S. Akdeniz 1337.

248. M. neglectum Guss., Y, Akçaören, wasteland, 850 m, 16.04.2009, S. Akdeniz 1336.

249. Bellevalia clusiana Griseb., Y, wasteland, 850 m, 23.04.2008, S. Akdeniz 1304. Irano - Turanian. Endemic. LC.

36. CYPERACEAE

250. Carex tomentosa L., A, Mountain pass of Ayaş, wasteland, 1060 m, 15.05.2009, S. Akdeniz 1354. Euro Siberian.

\section{POACEAE (GRAMINEAE)}

251. Aegilops speltoides Tausch. var. speltoides, A, Mountain pass of Ayaş, wasteland, 1060 m, 15.05.2009, S. Akdeniz 1297.

252. A. triuncialis L. subsp. triuncialis, A, Mountain pass of Ayaş, wasteland, $1060 \mathrm{~m}, 15.05 .2009$, S. Akdeniz 1352.

253. Hordeum geniculatum All., A, stony slopes, 980 m, 28.05.2008, S. Akdeniz 1345. Euro - Siberian.

254. H. murinum L. subsp. glaucum (Steudel) Tzvelev, Y, roadside, 855 m, 31.05.2009, S. Akdeniz 1299.

255. Bromus japonicus Thunb. subsp.japonicus, A, roadside, 950 m, 28.05.2008, S. Akdeniz 1293.

256. B. tectorum L., A, stony slopes, 980 m, 28.05.2008, S. Akdeniz 1292.

257. B. sterilis L., Y, roadside, 855 m, 31.05.2009, S. Akdeniz 1291.

258. B. madritensis L., Y, roadside, 855 m, 31.05.2009, S. Akdeniz 1296.

259. B. tomentellus Boiss., A, Mountain pass of Ayaş, wasteland, 1060 m, 15.05.2009, 1294. Irano - Turanian.

260. B. riparius Rehm., A, Mountain pass of Ayaş, wasteland, 1060 m, 15.05.2009, S. Akdeniz 1295.

261. Arrhenatherum elatius (L.) P. Beauv., Y, roadside, 855 m, 31.05.2009, S. Akdeniz 1344. Euro - Siberian.

262. Phleum pratense L., A, stony slopes, 980 m, 28.05.2008, S. Akdeniz 1301. Euro - Siberian.

263. P. montanum C. Koch. subsp. montanum, Y, roadside, 855 m, 31.05.2009, S. Akdeniz 1300.

264. Alopecurus myosuroides Hudson var. myosuroides, A, Mountain pass of Ayaş, wasteland, $1060 \mathrm{~m}$, 15.05.2009, S. Akdeniz 1353. Euro - Siberian.

265. Poa pratensis L., A, Mountain pass of Ayaş, wasteland, 1060 m, 15.05.2009, S. Akdeniz 1303.

266. P. angustifolia L., A, Mountain pass of Ayaş, wasteland, 1060 m, 15.05.2009, S. Akdeniz 1302.

267. P. timoleontis Heldr. ex Boiss., A, Mountain pass of Ayaş, wasteland, 1060 m, 15.05.2009, S. Akdeniz 1343. East Mediterranean.

268. P. bulbosa L., A, Mountain pass of Ayaş, wasteland, 1060 m, 15.05.2009, S. Akdeniz 1298. 